Network Working Group

Request for Comments: 5562

Category: Experimental
A. Kuzmanovic

A. Mondal

Northwestern University

S. Floyd

ICSI

K. Ramakrishnan

AT\&T Labs Research

June 2009

\title{
Adding Explicit Congestion Notification (ECN) Capability to TCP's SYN/ACK Packets
}

Status of This Memo

This memo defines an Experimental Protocol for the Internet community. It does not specify an Internet standard of any kind. Discussion and suggestions for improvement are requested. Distribution of this memo is unlimited.

Copyright Notice

Copyright (c) 2009 IETF Trust and the persons identified as the document authors. All rights reserved.

This document is subject to BCP 78 and the IETF Trust's Legal Provisions Relating to IETF Documents in effect on the date of publication of this document (http://trustee.ietf.org/license-info). Please review these documents carefully, as they describe your rights and restrictions with respect to this document.

This document may contain material from IETF Documents or IETF Contributions published or made publicly available before November 10, 2008. The person(s) controlling the copyright in some of this material may not have granted the IETF Trust the right to allow modifications of such material outside the IETF Standards Process. Without obtaining an adequate license from the person(s) controlling the copyright in such materials, this document may not be modified outside the IETF Standards Process, and derivative works of it may not be created outside the IETF Standards Process, except to format it for publication as an RFC or to translate it into languages other than English. 


\section{Abstract}

The proposal in this document is Experimental. While it may be deployed in the current Internet, it does not represent a consensus that this is the best possible mechanism for the use of Explicit Congestion Notification (ECN) in TCP SYN/ACK packets.

This document describes an optional, experimental modification to RFC 3168 to allow TCP SYN/ACK packets to be ECN-Capable. For TCP, RFC 3168 specifies setting an ECN-Capable codepoint on data packets, but not on SYN and SYN/ACK packets. However, because of the high cost to the TCP transfer of having a SYN/ACK packet dropped, with the resulting retransmission timeout, this document describes the use of ECN for the SYN/ACK packet itself, when sent in response to a SYN packet with the two ECN flags set in the TCP header, indicating a willingness to use ECN. Setting the initial TCP SYN/ACK packet as ECN-Capable can be of great benefit to the TCP connection, avoiding the severe penalty of a retransmission timeout for a connection that has not yet started placing a load on the network. The TCP responder (the sender of the SYN/ACK packet) must reply to a report of an ECNmarked SYN/ACK packet by resending a SYN/ACK packet that is not ECNCapable. If the resent SYN/ACK packet is acknowledged, then the TCP responder reduces its initial congestion window from two, three, or four segments to one segment, thereby reducing the subsequent load from that connection on the network. If instead the SYN/ACK packet is dropped, or for some other reason the TCP responder does not receive an acknowledgement in the specified time, the TCP responder follows TCP standards for a dropped SYN/ACK packet (setting the retransmission timer). 
Table of Contents

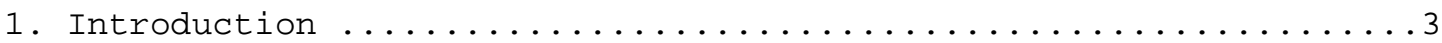

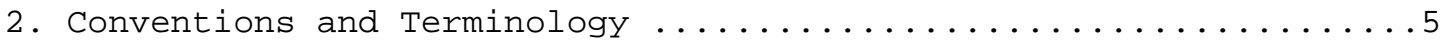

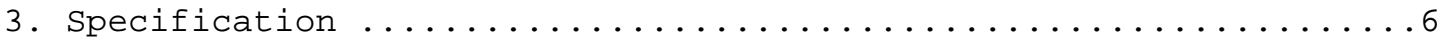

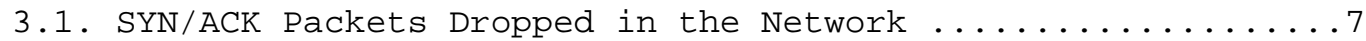

3.2. SYN/ACK Packets ECN-Marked in the Network ............8

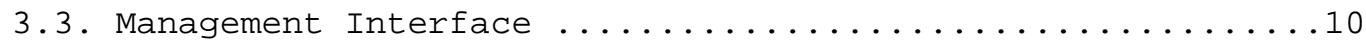

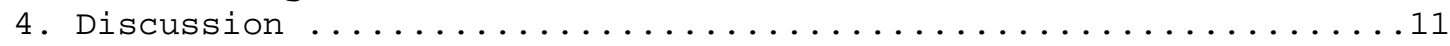

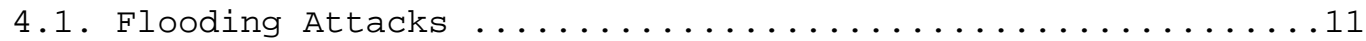

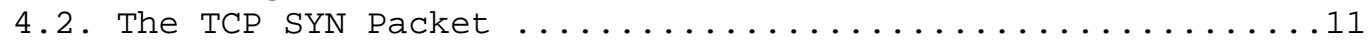

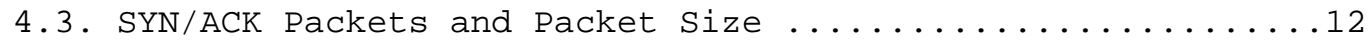

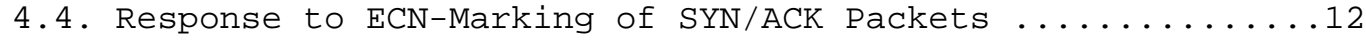

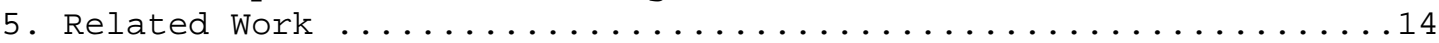

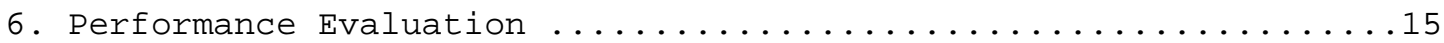

6.1. The Costs and Benefits of Adding ECN-Capability .......15

6.2. An Evaluation of Different Responses to ECN-Marked

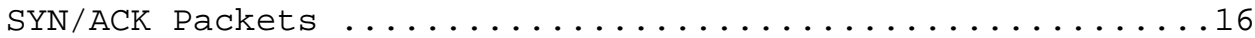

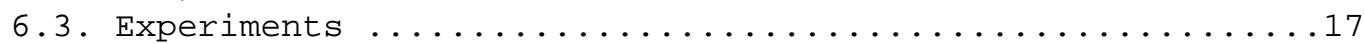

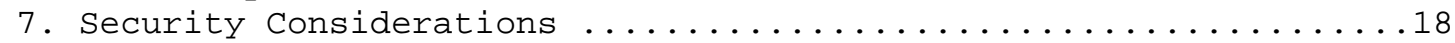

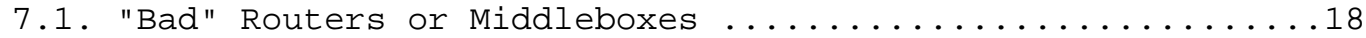

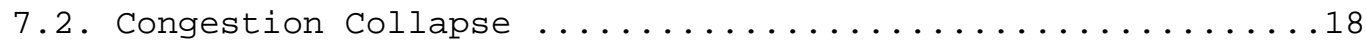

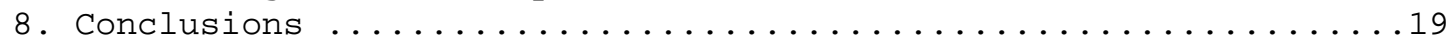

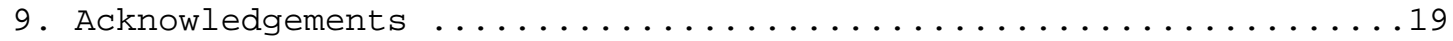

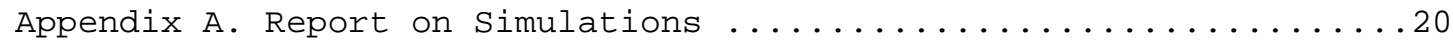

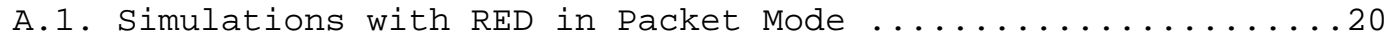

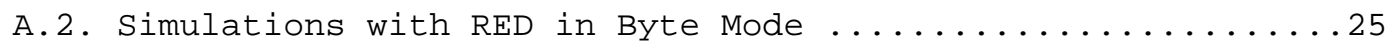

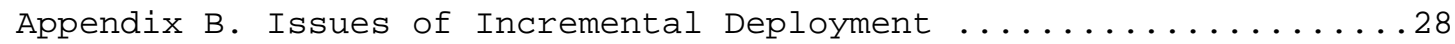

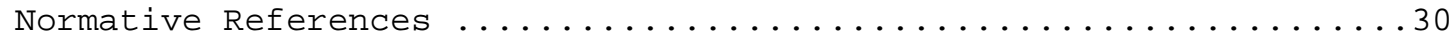

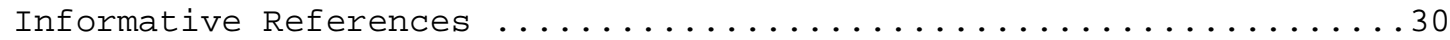

\section{Introduction}

TCP's congestion control mechanism has primarily used packet loss as the congestion indication, with packets dropped when buffers overflow. With such tail-drop mechanisms, the packet delay can be high, as the queue at bottleneck routers can be fairly large. Dropping packets only when the queue overflows, and having TCP react only to such losses, results in:

1) significantly higher packet delay;

2) unnecessarily many packet losses; and

3) unfairness due to synchronization effects. 
The adoption of Active Queue Management (AQM) mechanisms allows better control of bottleneck queues [RFC2309]. This use of AQM has the following potential benefits:

1) better control of the queue, with reduced queuing delay;

2) fewer packet drops; and

3) better fairness because of fewer synchronization effects.

With the adoption of ECN, performance may be further improved. When the router detects congestion before buffer overflow, the router can provide a congestion indication either by dropping a packet or by setting the Congestion Experienced ( $\mathrm{CE}$ ) codepoint in the Explicit Congestion Notification (ECN) field in the IP header [RFC3168]. The IETF has standardized the use of the Congestion Experienced (CE) codepoint in the IP header for routers to indicate congestion. For incremental deployment and backwards compatibility, the RFC on ECN [RFC3168] specifies that routers may mark ECN-Capable packets that would otherwise have been dropped, using the Congestion Experienced codepoint in the ECN field. The use of ECN allows TCP to react to congestion while avoiding unnecessary retransmission timeouts. Thus, using ECN has several benefits:

1) For short transfers, a TCP connection's congestion window may be small. For example, if the current window contains only one packet, and that packet is dropped, TCP will have to wait for a retransmission timeout to recover, reducing its overall throughput. Similarly, if the current window contains only a few packets and one of those packets is dropped, there might not be enough duplicate acknowledgements for a fast retransmission, and the sender of the data packet might have to wait for a delay of several round-trip times (RTT) using Limited Transmit [RFC3042]. With the use of ECN, short flows are less likely to have packets dropped, sometimes avoiding unnecessary delays or costly retransmission timeouts.

2) While longer flows may not see substantially improved throughput with the use of ECN, they may experience lower loss. This may benefit TCP applications that are latency- and loss-sensitive, because of the avoidance of retransmissions.

RFC 3168 [RFC3168] specifies setting the ECN-Capable codepoint on TCP data packets, but not on TCP SYN and SYN/ACK packets. RFC 3168 [RFC3168] specifies the negotiation of the use of ECN between the two TCP endpoints in the TCP SYN and SYN-ACK exchange, using flags in the TCP header. Erring on the side of being conservative, RFC 3168 [RFC3168] does not specify the use of ECN for the first SYN/ACK 
packet itself. However, because of the high cost to the TCP transfer of having a SYN/ACK packet dropped, with the resulting retransmission timeout, this document specifies the use of ECN for the SYN/ACK packet itself. This can be of great benefit to the TCP connection, avoiding the severe penalty of a retransmission timeout for a connection that has not yet started placing a load on the network. The sender of the SYN/ACK packet must respond to a report of an ECNmarked SYN/ACK packet (a SYN/ACK packet with the CE codepoint set in the ECN field in the IP header) by sending a non-ECN-Capable SYN/ACK packet, and by reducing its initial congestion window from two, three, or four segments to one segment, reducing the subsequent load from that connection on the network.

The use of ECN for SYN/ACK packets has the following potential benefits:

1) Avoidance of a retransmission timeout;

2) Improvement in the throughput of short connections.

This document specifies a modification to RFC 3168 [RFC3168] to allow TCP SYN/ACK packets to be ECN-Capable. Section 3 contains the specification of the change, while Section 4 discusses some of the issues, and Section 5 discusses related work. Section 6 contains an evaluation of the specified change.

2. Conventions and Terminology

The key words "MUST", "MUST NOT", "REQUIRED", "SHALL", "SHALL NOT", "SHOULD", "SHOULD NOT", "RECOMMENDED", "MAY", and "OPTIONAL" in this document are to be interpreted as described in [RFC2119].

We use the following terminology from RFC 3168 [RFC3168]:

The ECN field in the IP header:

O CE: the Congestion Experienced codepoint; and

- ECT: either one of the two ECN-Capable Transport codepoints.

The ECN flags in the TCP header:

o CWR: the Congestion Window Reduced flag; and

O ECE: the ECN-ECho flag. 
ECN-setup packets:

- ECN-setup SYN packet: a SYN packet with the ECE and CWR flags;

- ECN-setup SYN-ACK packet: a SYN-ACK packet with ECE but not CWR.

In this document, we use the terms "initiator" and "responder" to refer to the sender of the SYN packet and of the SYN-ACK packet, respectively.

3. Specification

This section specifies the modification to RFC 3168 [RFC3168] to allow TCP SYN/ACK packets to be ECN-Capable.

Section 6.1.1 of RFC 3168 [RFC3168] states that "A host MUST NOT set ECT on SYN or SYN-ACK packets". In this section, we specify that a TCP node may respond to an initial ECN-setup SYN packet by setting $\mathrm{ECT}$ in the responding ECN-setup SYN/ACK packet, indicating to routers that the SYN/ACK packet is ECN-Capable. This allows a congested router along the path to mark the packet instead of dropping the packet as an indication of congestion.

Assume that TCP node A transmits to TCP node B an ECN-setup SYN packet, indicating willingness to use ECN for this connection. As specified by RFC 3168 [RFC3168], if TCP node B is willing to use ECN, node $B$ responds with an ECN-setup SYN-ACK packet. 


\subsection{SYN/ACK Packets Dropped in the Network}

Figure 1 shows an interchange with the SYN/ACK packet dropped by a congested router. Node $B$ waits for a retransmission timeout, and then retransmits the SYN/ACK packet.

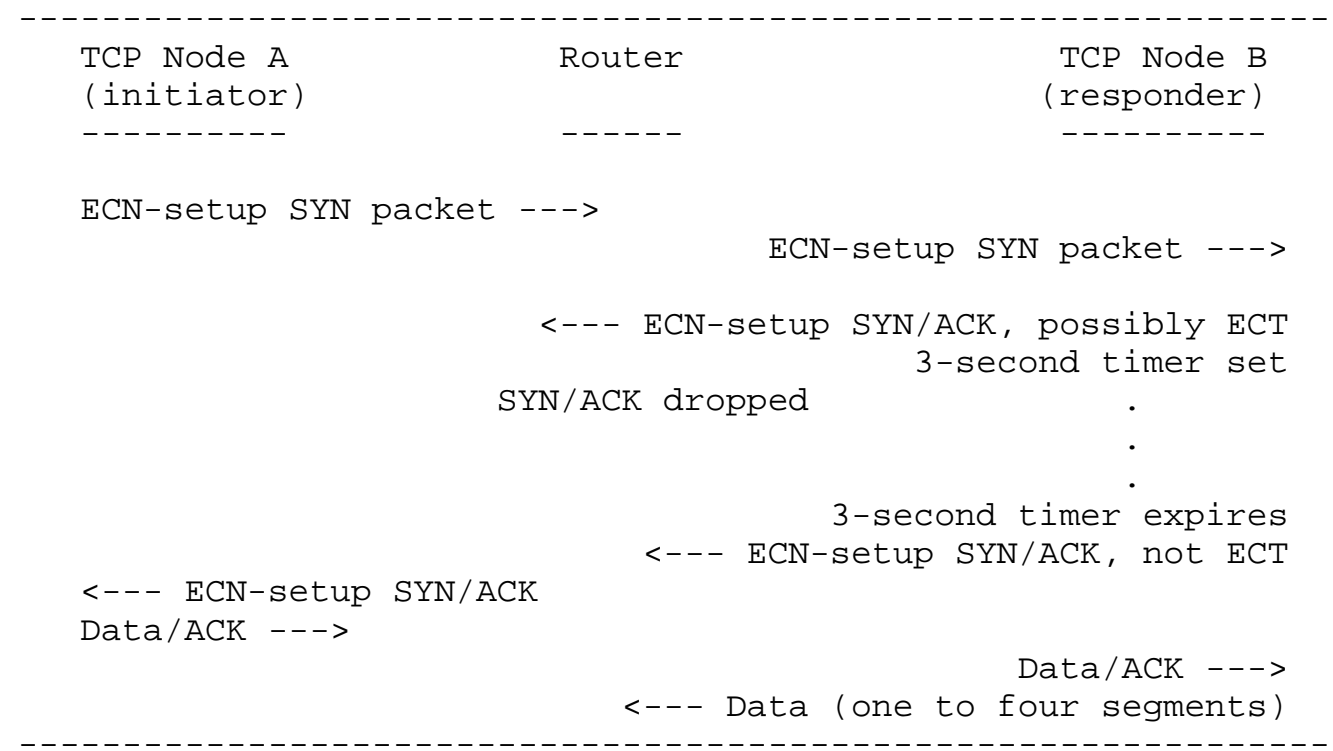

Figure 1: SYN exchange with the SYN/ACK packet dropped

If the SYN/ACK packet is dropped in the network, the responder (node B) responds by waiting three seconds for the retransmission timer to expire [RFC2988]. If a SYN/ACK packet with the ECT codepoint is dropped, the responder should resend the SYN/ACK packet without the ECN-Capable codepoint. (Although we are not aware of any middleboxes that drop SYN/ACK packets that contain an ECN-Capable codepoint in the IP header, we have learned to design our protocols defensively in this regard [RFC3360].)

We note that if syn-cookies were used by the responder (node B) in the exchange in Figure 1, the responder wouldn't set a timer upon transmission of the SYN/ACK packet [SYN-COOK] [RFC4987]. In this case, if the SYN/ACK packet was lost, the initiator (node A) would have to timeout and retransmit the SYN packet in order to trigger another SYN-ACK. 


\subsection{SYN/ACK Packets ECN-Marked in the Network}

Figure 2 shows an interchange with the SYN/ACK packet sent as ECNCapable, and ECN-marked instead of dropped at the congested router. This document specifies ECN+/TryOnce, which differs from the original proposal for ECN+ in [ECN+]; with ECN+/TryOnce, if the TCP responder is informed that the SYN/ACK was ECN-marked, the TCP responder immediately sends a SYN/ACK packet that is not ECN-Capable. The TCP responder is only allowed to send data packets after the TCP initiator reports the receipt of a SYN/ACK packet that is not ECNmarked.

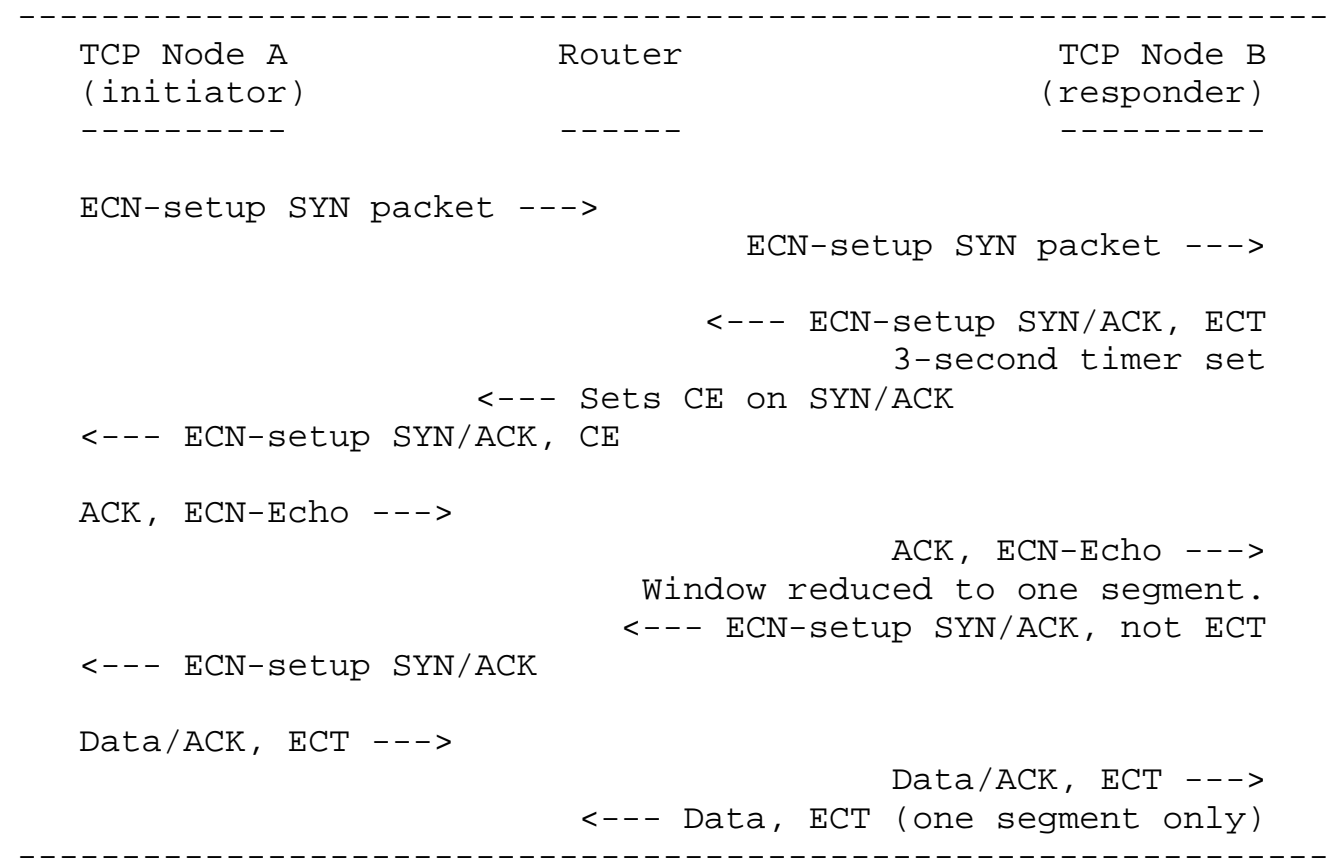

Figure 2: SYN exchange with the SYN/ACK packet marked $\mathrm{ECN}+/$ TryOnce

If the initiator (node A) receives a SYN/ACK packet that has been ECN-marked by the congested router, with the CE codepoint set, the initiator restarts the retransmission timer. The initiator responds to the ECN-marked SYN/ACK packet by setting the ECN-Echo flag in the TCP header of the responding ACK packet. The initiator uses the standard rules in setting the cumulative acknowledgement field in the responding ACK packet. 
The initiator does not advance from the "SYN-Sent" to the "Established" state until it receives a SYN/ACK packet that is not ECN-marked.

When the responder (node B) receives the ECN-Echo packet reporting the Congestion Experienced indication in the SYN/ACK packet, the responder sets the initial congestion window to one segment, instead of two segments as allowed by [RFC2581], or three or four segments allowed by [RFC3390]. As illustrated in Figure 2, if the responder (node B) receives an ECN-Echo packet informing it of a Congestion Experienced indication on its SYN/ACK packet, the responder sends a SYN/ACK packet that is not ECN-Capable, in addition to setting the initial window to one segment. The responder does not advance the send sequence number. The responder also sets the retransmission timer. The responder follows RFC 2988 [RFC2988] in setting the RTO (retransmission timeout).

The TCP hosts follow the standard specification for the response to duplicate SYN/ACK packets (e.g., Section 3.4 of RFC 793 [RFC793]).

We note that the mechanism in this document differs from RFC 3168 [RFC3168], which specifies that "the sending TCP MUST restart the retransmission timer on receiving the ECN-Echo packet when the congestion window is one". RFC 3168 [RFC3168] does not allow SYN/ACK packets to be ECN-Capable. RFC 3168 [RFC3168] specifies that in response to an ECN-Echo packet, the TCP responder also sets the CWR flag in the TCP header of the next data packet sent, to acknowledge its receipt of and reaction to the ECN-Echo flag. In contrast, in response to an ECN-Echo packet acknowledging the receipt of an ECNCapable SYN/ACK packet, the TCP responder doesn't set the CWR flag, but simply sends a SYN/ACK packet that is not ECN-Capable. On receiving the non-ECN-Capable SYN/ACK packet, the TCP initiator clears the ECN-Echo flag on replying packets. 


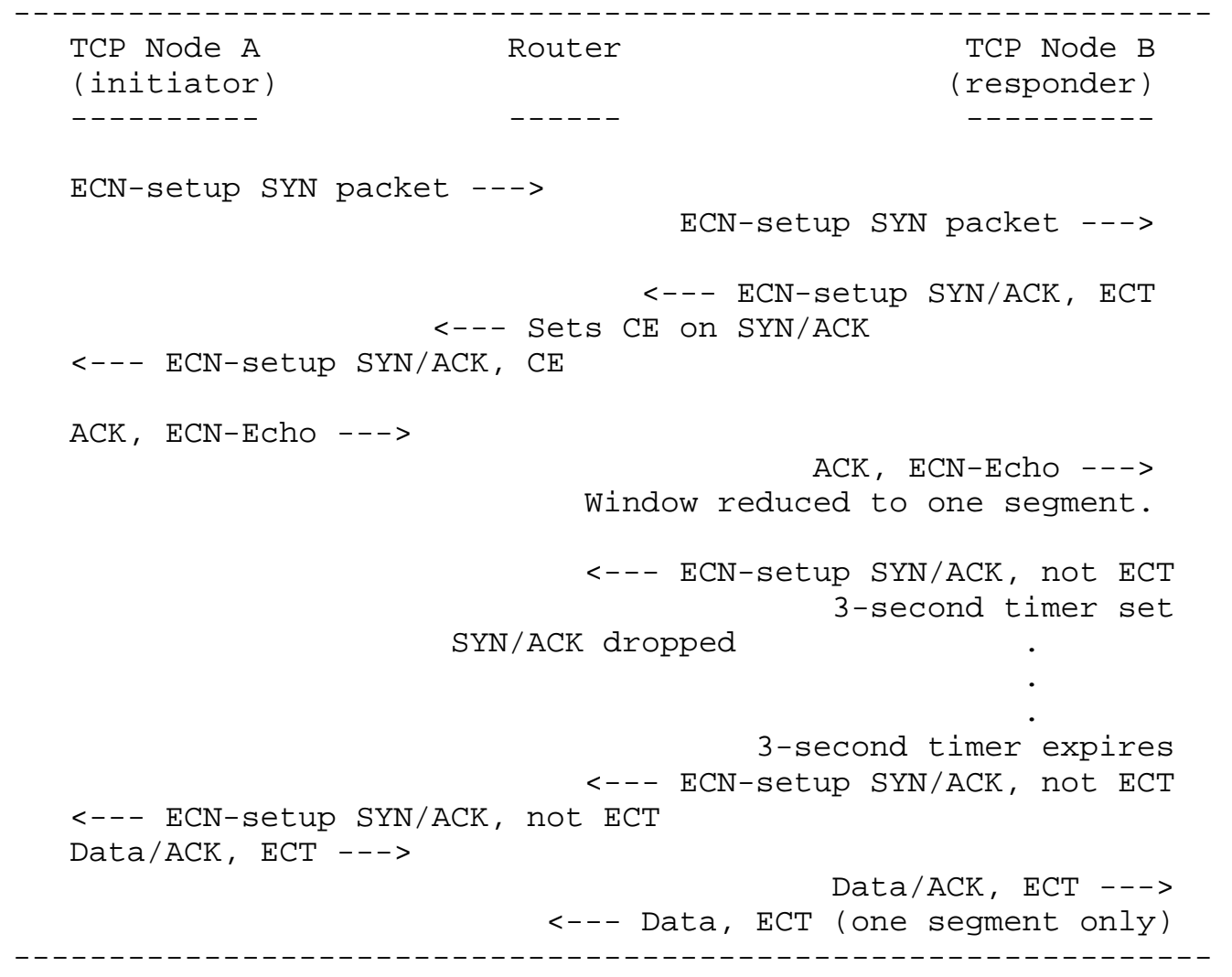

Figure 3: SYN exchange with the first SYN/ACK packet marked and the second SYN/ACK packet dropped - ECN+/TryOnce

In contrast to Figure 2, Figure 3 shows an interchange where the first SYN/ACK packet is ECN-marked and the second SYN/ACK packet is dropped in the network. As in Figure 2, the TCP responder sets a timer when the second SYN/ACK packet is sent. Figure 3 shows that if the timer expires before the TCP responder receives an acknowledgement for the other end, the TCP responder resends the SYN/ACK packet, following the TCP standards.

\subsection{Management Interface}

The TCP implementation using ECN-Capable SYN/ACK packets should include a management interface to allow the use of ECN to be turned off for SYN/ACK packets. This is to deal with possible backwards compatibility problems such as those discussed in Appendix B. 


\section{Discussion}

The rationale for the specification in this document is the following. When node B receives a TCP SYN packet with ECN-Echo bit set in the TCP header, this indicates that node A is ECN-Capable. If node $B$ is also ECN-Capable, there are no obstacles to immediately setting one of the ECN-Capable codepoints in the IP header in the responding TCP SYN/ACK packet.

There can be a great benefit in setting an ECN-Capable codepoint in SYN/ACK packets, as is discussed further in [ECN+], and reported briefly in section 5 below. Congestion is most likely to occur in the server-to-client direction. As a result, setting an ECN-Capable codepoint in SYN/ACK packets can reduce the occurrence of threesecond retransmission timeouts resulting from the drop of SYN/ACK packets.

\subsection{Flooding Attacks}

Setting an ECN-Capable codepoint in the responding TCP SYN/ACK packets does not raise any new or additional security vulnerabilities. For example, provoking servers or hosts to send SYN/ACK packets to a third party in order to perform a "SYN/ACK flood" attack would be highly inefficient. Third parties would immediately drop such packets, since they would know that they didn't generate the TCP SYN packets in the first place. Moreover, such SYN/ACK attacks would have the same signatures as the existing TCP SYN attacks. Provoking servers or hosts to reply with SYN/ACK packets in order to congest a certain link would also be highly inefficient because SYN/ACK packets are small in size.

However, the addition of ECN-Capability to SYN/ACK packets could allow SYN/ACK packets to persist for more hops along a network path before being dropped, thus adding somewhat to the ability of a SYN/ACK attack to flood a network link.

\subsection{The TCP SYN Packet}

There are several reasons why an ECN-Capable codepoint must not be set in the IP header of the initiating TCP SYN packet. First, when the TCP SYN packet is sent, there are no guarantees that the other TCP endpoint (node B in Figure 2) is ECN-Capable, or that it would be able to understand and react if the ECN CE codepoint was set by a congested router. 
Second, the ECN-Capable codepoint in TCP SYN packets could be misused by malicious clients to "improve" the well-known TCP SYN attack. BY setting an ECN-Capable codepoint in TCP SYN packets, a malicious host might be able to inject a large number of TCP SYN packets through a potentially congested ECN-enabled router, congesting it even further.

For both these reasons, we continue the restriction that the TCP SYN packet must not have the ECN-Capable codepoint in the IP header set.

\subsection{SYN/ACK Packets and Packet Size}

There are a number of router buffer architectures that have smaller dropping rates for small (SYN) packets than for large (data) packets. For example, for a Drop-Tail queue in units of packets, where each packet takes a single slot in the buffer regardless of packet size, small and large packets are equally likely to be dropped. However, for a Drop-Tail queue in units of bytes, small packets are less likely to be dropped than are large ones. Similarly, for Random Early Detection (RED) in packet mode, small and large packets are equally likely to be dropped or marked, while for RED in byte mode, a packet's chance of being dropped or marked is proportional to the packet size in bytes.

For a congested router with an AQM mechanism in byte mode, where a packet's chance of being dropped or marked is proportional to the packet size in bytes, the drop or marking rate for TCP SYN/ACK packets should generally be low. In this case, the benefit of making SYN/ACK packets ECN-Capable should be similarly moderate. However, for a congested router with a Drop-Tail queue in units of packets or with an $\mathrm{AQM}$ mechanism in packet mode, and with no priority queuing for smaller packets, small and large packets should have the same probability of being dropped or marked. In such a case, making SYN/ACK packets ECN-Capable should be of significant benefit.

We believe that there are a wide range of behaviors in the real world in terms of the drop or mark behavior at routers as a function of packet size (see Section 10 of [Tools]). We note that all of these alternatives listed above are available in the NS simulator (DropTail queues are by default in units of packets, while the default for RED queue management has been changed from packet mode to byte mode).

\subsection{Response to ECN-Marking of SYN/ACK Packets}

One question is why TCP SYN/ACK packets should be treated differently from other packets in terms of the end node's response to an ECNmarked packet. Section 5 of RFC 3168 [RFC3168] specifies the following: 
Upon the receipt by an ECN-Capable transport of a single CE packet, the congestion control algorithms followed at the endsystems MUST be essentially the same as the congestion control response to a *single* dropped packet. For example, for ECNCapable TCP the source TCP is required to halve its congestion window for any window of data containing either a packet drop or an ECN indication.

In particular, Section 6.1.2 of RFC 3168 [RFC3168] specifies that when the TCP congestion window consists of a single packet and that packet is ECN-marked in the network, then the data sender must reduce the sending rate below one packet per round-trip time, by waiting for one RTO before sending another packet. If the RTO was set to the average round-trip time, this would result in halving the sending rate; because the RTO is in fact larger than the average round-trip time, the sending rate is reduced to less than half of its previous value.

$\mathrm{TCP}^{\prime} \mathrm{S}$ congestion control response to the *dropping* of a SYN/ACK packet is to wait a default time before sending another packet. This document argues that ECN gives end-systems a wider range of possible responses to the *marking* of a SYN/ACK packet, and that waiting a default time before sending another packet is not the desired response.

On the conservative end, one could assume an effective congestion window of one packet for the SYN/ACK packet, and respond to an ECNmarked SYN/ACK packet by reducing the sending rate to one packet every two round-trip times. As an approximation, the TCP end node could measure the round-trip time $T$ between the sending of the SYN/ACK packet and the receipt of the acknowledgement, and reply to the acknowledgement of the ECN-marked SYN/ACK packet by waiting $T$ seconds before sending a data packet.

However, we note that for an ECN-marked SYN/ACK packet, halving the *congestion window* is not the same as halving the *sending rate*; there is no "sending rate" associated with an ECN-Capable SYN/ACK packet, as such packets are only sent as the first packet in a connection from that host. Further, a router's marking of a SYN/ACK packet is not affected by any past history of that connection.

Adding ECN-Capability to SYN/ACK packets allows the response of the responder setting the initial congestion window to one packet, instead of its allowed default value of two, three, or four packets. The responder sends a non-ECN-Capable SYN/ACK packet, and proceeds 
with a cautious sending rate of one data packet per round-trip time after that SYN/ACK packet is acknowledged. This document argues that this approach is useful to users, with no dangers of congestion collapse or of starvation of competing traffic. This is discussed in more detail below in section 6.2 .

We note that if the data transfer is entirely from node A to node B, there is still a difference in performance between the original mechanism ECN+ and the mechanism ECN+/TryOnce specified in this document. In particular, with ECN+/TryOnce, the TCP originator does not send data packets until it has received a non-ECN-marked SYN/ACK packet from the other end.

5. Related Work

The addition of ECN-Capability to TCP'S SYN/ACK packets was initially proposed in [ECN+]. The paper includes an extensive set of simulation and testbed experiments to evaluate the effects of the proposal, using several Active Queue Management (AQM) mechanisms, including Random Early Detection (RED) [RED], Random Exponential Marking (REM) [REM], and Proportional Integrator (PI) [PI]. The performance measures were the end-to-end response times for each request/response pair, and the aggregate throughput on the bottleneck link. The end-to-end response time was computed as the time from the moment when the request for the file is sent to the server, until that file is successfully downloaded by the client.

The measurements from [ECN+] show that setting an ECN-Capable codepoint in the IP packet header in TCP SYN/ACK packets systematically improves performance with all evaluated AQM schemes. When SYN/ACK packets at a congested router are ECN-marked instead of dropped, this can avoid a long initial retransmission timeout, improving the response time for the affected flow dramatically.

[ECN+] shows that the impact on aggregate throughput can also be quite significant, because marking SYN ACK packets can prevent larger flows from suffering long timeouts before being "admitted" into the network. In addition, the testbed measurements from [ECN+] show that web servers setting the ECN-Capable codepoint in TCP SYN/ACK packets could serve more requests.

As a final step, [ECN+] explores the coexistence of flows that do and don't set the ECN-Capable codepoint in TCP SYN/ACK packets. The results in $[\mathrm{ECN}+]$ show that both types of flows can coexist, with some performance degradation for flows that don't use ECN+. Flows 
that do use ECN+ improve their end-to-end performance. At the same time, the performance degradation for flows that don't use ECN+, as a result of the flows that do use ECN+, increases as a greater fraction of flows use ECN+.

6. Performance Evaluation

6.1. The Costs and Benefits of Adding ECN-Capability

[ECN+] explores the costs and benefits of adding ECN-Capability to SYN/ACK packets with both simulations and experiments. The addition of ECN-Capability to SYN/ACK packets could be of significant benefit for those ECN connections that would have had the SYN/ACK packet dropped in the network, and for which the ECN-Capability would allow the SYN/ACK to be marked rather than dropped.

The percent of SYN/ACK packets on a link can be quite high. In particular, measurements on links dominated by web traffic indicate that 15-20\% of the packets can be SYN/ACK packets [SCJO01].

The benefit of adding ECN-Capability to SYN/ACK packets depends in part on the size of the data transfer. The drop of a SYN/ACK packet can increase the download time of a short file by an order of magnitude, by requiring a three-second retransmission timeout. For longer-lived flows, the effect of a dropped SYN/ACK packet on file download time is less dramatic. However, even for longer-lived flows, the addition of ECN-Capability to SYN/ACK packets can improve the fairness among long-lived flows, as newly arriving flows would be less likely to have to wait for retransmission timeouts.

One question that arises is what fraction of connections would see the benefit from making SYN/ACK packets ECN-Capable in a particular scenario. Specifically:

(1) What fraction of arriving SYN/ACK packets are dropped at the congested router when the SYN/ACK packets are not ECN-Capable?

(2) Of those SYN/ACK packets that are dropped, what fraction would have been ECN-marked instead of dropped if the SYN/ACK packets had been ECN-Capable?

To answer (1), it is necessary to consider not only the level of congestion but also the queue architecture at the congested link. As described in section 4 above, for some queue architectures, small packets are less likely to be dropped than large ones. In such an environment, SYN/ACK packets would have lower packet drop rates; question (1) could not necessarily be inferred from the overall packet drop rate, but could be answered by measuring the drop rate 
for SYN/ACK packets directly. In such an environment, adding ECNCapability to SYN/ACK packets would be of less dramatic benefit than in environments where all packets are equally likely to be dropped regardless of packet size.

As question (2) implies, even if all of the SYN/ACK packets were ECN-Capable, there could still be some SYN/ACK packets dropped instead of marked at the congested link; the full answer to question (2) depends on the details of the queue management mechanism at the router. If congestion is sufficiently bad, and the queue management mechanism cannot prevent the buffer from overflowing, then SYN/ACK packets will be dropped rather than marked upon buffer overflow whether or not they are ECN-Capable.

For some AQM mechanisms, ECN-Capable packets are marked instead of dropped any time this is possible, that is, any time the buffer is not yet full. For other AQM mechanisms however, such as the RED mechanism as recommended in [RED], packets are dropped rather than marked when the packet drop/mark rate exceeds a certain threshold, e.g., 10\%, even if the packets are ECN-Capable. For a router with such an AQM mechanism, when congestion is sufficiently severe to cause a high drop/mark rate, some SYN/ACK packets would be dropped instead of marked whether or not they were ECN-Capable.

Thus, the degree of benefit of adding ECN-Capability to SYN/ACK packets depends not only on the overall packet drop rate in the network, but also on the queue management architecture at the congested link.

\subsection{An Evaluation of Different Responses to ECN-Marked SYN/ACK Packets}

This document specifies that the end node responds to the report of an ECN-marked SYN/ACK packet by setting the initial congestion window to one segment, instead of its possible default value of two to four segments, and resending a SYN/ACK packet that is not ECN-Capable. We call this ECN+/TryOnce.

However, Section 4 discussed two other possible responses to an ECNmarked SYN/ACK packet. In ECN+, the original proposal from [ECN+], the end node responds to the report of an ECN-marked SYN/ACK packet by setting the initial congestion window to one segment and immediately sending a data packet, if it has one to send. In ECN+/Wait, the end node responds to the report of an ECN-marked SYN/ACK packet by setting the initial congestion window to one segment and waiting an RTT before sending a data packet. 
Simulations comparing the performance with standard ECN (without ECN-marked SYN/ACK packets), ECN+, ECN+/Wait, and ECN/TryOnce show little difference, in terms of aggregate congestion, between ECN+ and ECN+/Wait. However, for some scenarios with queues that are packetbased rather than byte-based, and with packet drop rates above 25\% without ECN+, the use of ECN+ or of ECN+/Wait can more than double the packet drop rates to greater than 50\%. The details are given in Tables 1 and 3 of Appendix A below. ECN+/TryOnce does not increase the packet drop rate in scenarios of high congestion. Therefore, $\mathrm{ECN}+/$ TryOnce is superior to ECN+ or to ECN+/Wait, which both significantly increase the packet drop rate in scenarios of high congestion. At the same time, ECN+/Tryonce gives a performance improvement similar to that of ECN+ or ECN+/Wait (Tables 2 and 4 of Appendix A) .

Our conclusions are that ECN+/TryOnce is safe, and has significant benefits to the user, and avoids the problems of ECN+ or ECN+/Wait under extreme levels of congestion. As a consequence, this document specifies the use of ECN+/TryOnce.

Note: We only discovered the occasional congestion-related problems of ECN+ and of ECN+/Wait when re-running the simulations with an updated version of the ns-2 simulator, after the document had almost completed the standardization process.

\title{
6.3. Experiments
}

This section discusses experiments that would be useful before a widespread deployment of ECN-Capability for TCP SYN/ACK packets.

\begin{abstract}
Section 7.1 below discusses some of the known deployment problems of ECN, in terms of routers or middleboxes that react inappropriately to packets that use ECN codepoints in the IP or TCP packet headers. One goal of a measurement study of ECN-Capability for TCP SYN/ACK packets would be to determine if there were any routers or middleboxes that react inappropriately to TCP SYN/ACK packets containing an ECNCapable or CE codepoint in the IP header. A second goal of a measurement study would be to check the deployment status of older TCP implementations that are ECN-Capable, but that don't respond to ECN-Capability for SYN/ACK packets. (This is discussed in more detail in Appendix B below.)

Following the discussion in section 6.2, an experimental study could explore the use of ECN-Capability for TCP SYN/ACK packets in highly congested environments with ECN-Capable routers.
\end{abstract}




\section{Security Considerations}

TCP packets carrying the ECT codepoint in IP headers can be marked rather than dropped by ECN-Capable routers. This raises several security concerns that we discuss below.

\section{1. "Bad" Routers or Middleboxes}

There are a number of known deployment problems from using ECN with TCP traffic in the Internet. The first reported problem, dating back to 2000, is of a small but decreasing number of routers or middleboxes that reset a TCP connection in response to TCP SYN packets using flags in the TCP header to negotiate ECN-Capability [Kelson00] [RFC3360] [MAF05]. Dave Thaler reported at the March 2007 IETF of two new problems encountered by TCP connections using ECN; the first of the two problems concerns routers that crash when a TCP data packet arrives with the ECN field in the IP header with the codepoint ECT(0) or ECT(1), indicating that an ECN-Capable connection has been established [SBT07].

While there is no evidence that any routers or middleboxes drop SYN/ACK packets that contain an ECN-Capable or CE codepoint in the IP header, such behavior cannot be excluded. (There seems to be a number of routers or middleboxes that drop TCP SYN packets that contain known or unknown IP options (see figure 1 of [MAF05].) Thus, as specified in section 3, if a SYN/ACK packet with the ECT or CE codepoint is dropped, the TCP node should resend the SYN/ACK packet without the ECN-Capable codepoint. There is also no evidence that any routers or middleboxes crash when a SYN/ACK arrives with an ECNCapable or CE codepoint in the IP header (over and above the routers already known to crash when a data packet arrives with either ECT(0) or ECT(1)), but we have not conducted any measurement studies of this [F07].

\subsection{Congestion Collapse}

Because TCP SYN/ACK packets carrying an ECT codepoint could be ECNmarked instead of dropped at an ECN-Capable router, the concern is whether this can either invoke congestion or worsen performance in highly congested scenarios. However, after learning that a SYN/ACK packet was ECN-marked, the responder sends a SYN/ACK packet that is not ECN-Capable; if this SYN/ACK packet is dropped, the responder then waits for a retransmission timeout, as specified in the TCP standards. In addition, routers are free to drop rather than mark arriving packets in times of high congestion, regardless of whether the packets are ECN-Capable. When congestion is very high and a router's buffer is full, the router has no choice but to drop rather than to mark an arriving packet. 
The simulations reported in Appendix A show that even with demanding traffic mixes dominated by short flows and high levels of congestion, the aggregate packet dropping rates are not significantly different with Standard ECN or with ECN+/TryOnce. However, in our simulations, we have one scenario where ECN+ or ECN+/Wait results in a significantly higher packet drop rate than ECN or ECN+/TryOnce (Tables 1 and 3 in Appendix A below).

\section{Conclusions}

This document specifies a modification to RFC 3168 [RFC3168] to allow TCP nodes to send SYN/ACK packets as being ECN-Capable. Making the SYN/ACK packet ECN-Capable avoids the high cost to a TCP transfer when a SYN/ACK packet is dropped by a congested router, by avoiding the resulting retransmission timeout. This improves the throughput of short connections. This document specifies the ECN+/Tryonce mechanism for ECN-Capability for SYN/ACK packets, where the sender of the SYN/ACK packet responds to an ECN mark by reducing its initial congestion window from two, three, or four segments to one segment, and sending a SYN/ACK packet that is not ECN-Capable. The addition of ECN-Capability to SYN/ACK packets is particularly beneficial in the server-to-client direction, where congestion is more likely to occur. In this case, the initial information provided by the ECN marking in the SYN/ACK packet enables the server to appropriately adjust the initial load it places on the network, while avoiding the delay of a retransmission timeout.

9. Acknowledgements

We thank Anil Agarwal, Mark Allman, Remi Denis-Courmont, Wesley Eddy, Lars Eggert, Alfred Hoenes, Janardhan Iyengar, and Pasi Sarolahti for feedback on earlier working drafts of this document. We thank Adam Langley [L08] for contributing a patch for ECN+/TryOnce for the Linux development tree. 
Appendix A. Report on Simulations

This section reports on simulations showing the costs of adding ECN+ in highly congested scenarios. This section also reports on simulations for a comparative evaluation between ECN, ECN+, $\mathrm{ECN}+/$ Wait, and ECN+/TryOnce.

The simulations are run with a range of file-size distributions, using the PackMime traffic generator in the ns-2 simulator. They all use a heavy-tailed distribution of file sizes. The simulations reported in the tables below use a mean file size of 3 Kbytes, to show the results with a traffic mix with a large number of small transfers. Other simulations were run with mean file sizes of 5 Kbytes, 7 Kbytes, 14 Kbytes, and 17 Kbytes. The title of each chart gives the targeted average load from the traffic generator. Because the simulations use a heavy-tailed distribution of file sizes, and run for only 85 seconds (including ten seconds of warm-up time), the actual load is often much smaller than the targeted load. The congested link is $100 \mathrm{Mbps}$. RED is run in gentle mode, and arriving ECN-Capable packets are only dropped instead of marked if the buffer is full (and the router has no choice).

We explore three possible mechanisms for a TCP node's response to a report of an ECN-marked SYN/ACK packet. With ECN+, the TCP node sends a data packet immediately (with an initial congestion window of one segment). With ECN+/Wait, the TCP node waits a round-trip time before sending a data packet; the responder already has one measurement of the round-trip time when the acknowledgement for the SYN/ACK packet is received. With ECN+/TryOnce, the mechanism standardized in this document, the TCP responder replies to a report of an ECN-marked SYN/ACK packet by sending a SYN/ACK packet that is not ECN-Capable, and reducing the initial congestion window to one segment.

The simulation scripts are available on [ECN-SYN], along with graphs showing the distribution of response times for the TCP connections.

\section{A.1. Simulations with RED in Packet Mode}

The simulations with RED in packet mode and with the queue in packets show that ECN+ is useful in times of moderate or high congestion. However, for the simulations with a target load of 125\%, with a packet loss rate of over 25\% for ECN, ECN+ and ECN+/Wait both result in a packet loss rate of over 50\%. (In contrast, the packet loss rate with ECN+/Tryonce is less than that of ECN alone.) For the distribution of response times, the simulations show that ECN+, ECN+/Wait, and ECN+/TryOnce all significantly improve the response times, when compared to the response times with standard ECN. 
Table 1 shows the congestion levels for simulations with RED in packet mode, with a queue in packets. To explore a worst-case scenario, these simulations use a traffic mix with an unrealistically small flow size distribution, with a mean flow size of 3 kbytes. For each table showing a particular traffic load, the four rows show the number of packets dropped, the number of packets ECN-marked, the aggregate packet drop rate, and the aggregate throughput. The four columns show the simulations with standard ECN, ECN+, ECN+/Wait, and $\mathrm{ECN}+/$ TryOnce.

These simulations were run with RED set to mark instead of drop packets any time that the queue is not full. This is a worst-case scenario for ECN+ and its variants. For the default implementation of RED in the ns-2 simulator, when the average queue size exceeds a configured threshold, the router drops all arriving packets. For scenarios with this RED mechanism, it is less likely that ECN+ or one of its variants would increase the average queue size above the configured threshold.

The usefulness of ECN+: The first thing to observe is that for all of the simulations, the use of ECN+ or ECN+/Wait significantly increases the number of packets marked. In contrast, the use of ECN+/Tryonce significantly increases the number of packets marked in the simulations with moderate congestion, and gives a more moderate increase in the number of packets marked for the simulations with higher levels of congestion. However, the cumulative distribution function (CDF) in Table 2 shows that ECN+, ECN+/Wait, and ECN+/TryOnce all improve response times for all of the simulations, with moderate or with larger levels of congestion.

Little increase in congestion, sometimes: The second thing to observe is that for the simulations with low or moderate levels of congestion (that is, with packet drop rates less than $10 \%$ ), the use of $\mathrm{ECN}+$, ECN+/Wait, and ECN+/TryOnce all decrease the aggregate packet drop rate relative to the simulations with ECN. This makes sense, since with low or moderate levels of congestion, ECN+ allows SYN/ACK packets to be marked instead of dropped, and the use of ECN+ doesn't add to the aggregate congestion. However, for the simulations with packet drop rates of $15 \%$ or higher with ECN, the use of ECN+ or $\mathrm{ECN}+$ /Wait increases the aggregate packet drop rate, sometimes even doubling it.

Comparing ECN+, ECN+/Wait, and ECN+/TryOnce: The aggregate packet drop rate is generally higher with ECN+/Wait than with ECN+. Thus, there is no congestion-related reason to prefer ECN+/Wait over ECN+. In contrast, the aggregate packet drop rate with ECN+/TryOnce is often significantly lower than the aggregate packet drop rate with either ECN, ECN+, or ECN+/Wait. 


\begin{tabular}{|c|c|c|c|c|}
\hline & $\mathrm{ECN}$ & $\mathrm{ECN}+$ & $\mathrm{ECN}+/$ Wait & $\mathrm{ECN}+/$ TryOnce \\
\hline & --- & -------- & -------- & --------- \\
\hline Dropped & 20,516 & 11,226 & 11,735 & $16,755^{\prime}$ \\
\hline Marked & 30,586 & 37,741 & 37,425 & 40,764 \\
\hline Loss rate & $1.41 \%$ & $0.78 \%$ & $0.81 \%$ & $1.02 \%$ \\
\hline Throughput & $81 \%$ & $81 \%$ & $81 \%$ & $81 \%$ \\
\hline Target Loa & $\mathrm{ad}=110 \%:$ & & & \\
\hline & $\mathrm{ECN}$ & $\mathrm{ECN}+$ & $\mathrm{ECN}+/$ Wait & $\mathrm{ECN}+/$ TryOnce \\
\hline & ------- & ------- & ------- & ---------- \\
\hline Dropped & 165,566 & 106,083 & 147,180 & 208,422 \\
\hline Marked & 179,735 & 281,306 & 308,473 & 235,483 \\
\hline Loss rate & $9.01 \%$ & $6.12 \%$ & $8.02 \%$ & $6.89 \%$ \\
\hline Throughput & $92 \%$ & $92 \%$ & $92 \%$ & $94 \%$ \\
\hline Target Loa & $\mathrm{ad}=125 \%:$ & & & \\
\hline & $\mathrm{ECN}$ & $\mathrm{ECN}+$ & $\mathrm{ECN}+/$ Wait & $\mathrm{ECN}+/$ TryOnce \\
\hline & -------- & ------- & ------- & --------- \\
\hline Dropped & 600,628 & $1,746,768$ & $2,176,530$ & 625,552 \\
\hline Marked & 418,433 & $1,166,450$ & $1,164,932$ & 439,847 \\
\hline Loss rate & $25.45 \%$ & $51.73 \%$ & $56.87 \%$ & $18.31 \%$ \\
\hline Throughput & $94 \%$ & $98 \%$ & $97 \%$ & $95 \%$ \\
\hline Target Loa & $150 \%$ & & & \\
\hline & $\mathrm{ECN}$ & $\mathrm{ECN}+$ & $\mathrm{ECN}+/$ Wait & $\mathrm{ECN}+/$ TryOnce \\
\hline & ------- & $\begin{array}{l}------- \\
-10\end{array}$ & ------- & --------1 \\
\hline Dropped & $1,449,945$ & $1,565,0517$ & $1,563,0801$ & $1,351,637$ \\
\hline Marked & 669,840 & 583,378 & 591,315 & 684,715 \\
\hline Loss rate & $46.7 \%$ & $59.0 \%$ & $59.0 \%$ & $32.7 \%$ \\
\hline Throughput & $88 \%$ & $94 \%$ & $94 \div$ & $92 \%$ \\
\hline
\end{tabular}

Table 1: Simulations with an average flow size of 3 Kbytes, a 100 Mbps link, RED in packet mode, queue in packets 


\begin{tabular}{|c|c|c|c|c|c|c|c|c|c|c|c|}
\hline TI & 10 & 100 & 200 & 300 & 400 & 500 & 1000 & 2000 & 3000 & 4000 & 5000 \\
\hline $\mathrm{CN}$ : & 0.00 & 0.07 & 0.26 & 0.51 & 0.82 & 0.96 & 0.97 & 0.97 & 0.97 & 1.00 & 1.00 \\
\hline $\mathrm{CN}+:$ & 0.00 & 0.07 & 0.27 & 0.53 & 0.85 & 0.99 & 1.00 & 1.00 & 1.00 & 1.00 & 1.00 \\
\hline Wait: & 0.00 & 0.07 & 0.26 & 0.51 & 0.83 & 0.97 & 1.00 & 1.00 & 1.00 & 1.00 & 1.00 \\
\hline Once: & 0.00 & 0.07 & 0.24 & 0.49 & 0.83 & 0.97 & 1.00 & 1.00 & 1.00 & 1.00 & 1.00 \\
\hline arget & oad & $=11$ & & & & & & & & & \\
\hline TIME : & 10 & 100 & 200 & 300 & 400 & 500 & 1000 & 2000 & 3000 & 4000 & 5000 \\
\hline $\mathrm{CN}$ & & $\Omega$ & 19 & 11 & 07 & 19 & & 20 & & & 96 \\
\hline $\mathrm{ECN}+$ : & 0.00 & 0.07 & 0.22 & $\begin{array}{l}0.41 \\
0.48\end{array}$ & 0.81 & 0.96 & 1.00 & 1.00 & 1.00 & $\begin{array}{l}0.96 \\
1.00\end{array}$ & $\begin{array}{l}.96 \\
1.00\end{array}$ \\
\hline Wait: & 0.00 & 0.05 & 0.18 & 0.38 & 0.64 & 0.77 & 0.95 & 1.00 & 1.00 & 1.00 & 1.00 \\
\hline Once: & 0.00 & 0.06 & 0.19 & 0.42 & 0.70 & 0.86 & 0.95 & 0.96 & 0.96 & 0.99 & 0.99 \\
\hline Target & oad & $=12$ & & & & & & & & & \\
\hline TIME : & 10 & 100 & 200 & 300 & 400 & 500 & 1000 & 2000 & 3000 & 4000 & 5000 \\
\hline $\mathrm{ECN}$ : & 0.00 & 0.04 & 0.13 & 0.27 & 0.46 & 0.56 & 0.58 & 0.59 & 0.59 & 0.82 & 0.82 \\
\hline $\mathrm{ECN}+$ : & 0.00 & 0.06 & 0.18 & 0.33 & 0.58 & 0.76 & 0.97 & 0.99 & 0.99 & 1.00 & 1.00 \\
\hline Wait: & 0.00 & 0.01 & 0.06 & 0.13 & 0.21 & 0.27 & 0.68 & 0.98 & 0.99 & 1.00 & 1.00 \\
\hline Once: & 0.00 & 0.05 & 0.16 & 0.34 & 0.58 & 0.73 & 0.85 & 0.87 & 0.87 & 0.95 & 0.96 \\
\hline Target & bad & $=15$ & $5:$ & & & & & & & & \\
\hline TIME : & 10 & 100 & 200 & 300 & 400 & 500 & 1000 & 2000 & 3000 & 4000 & 5000 \\
\hline $\mathrm{ECN}$ : & 0. & 0.03 & 0.08 & 0.18 & 0.31 & 0.39 & 0.42 & & 0.43 & 0.68 & 0.68 \\
\hline $\mathrm{ECN}+$ : & 0.00 & 0.06 & 0.18 & 0.39 & 0.67 & 0.81 & 0.83 & 0.84 & 0.84 & 0.93 & 0.93 \\
\hline Wait: & 0.00 & 0.06 & 0.18 & 0.39 & 0.67 & 0.81 & 0.83 & 0.84 & 0.84 & 0.93 & 0.94 \\
\hline Once: & 0.00 & 0.04 & 0.13 & 0.27 & 0.46 & 0.59 & 0.72 & 0.75 & 0.75 & 0.88 & 0.88 \\
\hline e 2: & \multicolumn{11}{|c|}{$\begin{array}{l}\text { The cumulative distribution function (CDF) for transfer } \\
\text { times, for simulations with an average flow size of } 3 \\
\text { Kbytes, a } 100 \text { Mbps link, RED in packet mode, queue in } \\
\text { packets (the graphs are available from } \\
\text { "http://www.icir.org/floyd/ecn-syn/") }\end{array}$} \\
\hline
\end{tabular}




\begin{tabular}{|c|c|c|c|c|}
\hline & $\begin{array}{c}\mathrm{ECN} \\
-------\end{array}$ & $\begin{array}{c}\mathrm{ECN}+ \\
-------\end{array}$ & $\begin{array}{c}\mathrm{ECN}+/ \text { Wait } \\
-------\end{array}$ & $\begin{array}{c}\mathrm{ECN}+/ \operatorname{TryOnce} \\
---------\end{array}$ \\
\hline Dropped & 8,448 & 6,362 & 7,740 & 14,107 \\
\hline Marked & 9,891 & 16,787 & 17,456 & 16,132 \\
\hline Loss rate & $5.5 \%$ & $4.3 \%$ & $5.0 \%$ & $5.0 \%$ \\
\hline Throughput & $78 \%$ & $78 \%$ & $78 \%$ & $81 \%$ \\
\hline \multirow[t]{3}{*}{ Target Load } & $=110 \%$ & & & \\
\hline & $\mathrm{ECN}$ & $\mathrm{ECN}+$ & ECN+/Wait & $\mathrm{ECN}+/$ TryOnce \\
\hline & ------- & ------- & ------- & ---------- \\
\hline Dropped & 31,284 & 29,773 & 49,297 & 45,277 \\
\hline Marked & 28,429 & 54,729 & 60,383 & 34,622 \\
\hline Loss rate & $15.3 \%$ & $15.2 \%$ & $21.9 \%$ & $13.6 \%$ \\
\hline Throughput & $97 \%$ & $96 \%$ & $96 \%$ & $94 \%$ \\
\hline \multirow[t]{3}{*}{ Target Load } & $=125 \%$ & & & \\
\hline & $\mathrm{ECN}$ & $\mathrm{ECN}+$ & ECN+/Wait & $\mathrm{ECN}+/ \operatorname{TryOnce}$ \\
\hline & -------- & ------- & -------- & ---------- \\
\hline Dropped & 61,433 & 176,682 & 214,096 & 75,612 \\
\hline Marked & 44,408 & 119,728 & 117,301 & 49,442 \\
\hline Loss rate & $25.4 \%$ & $51.9 \%$ & $56.0 \%$ & $22.3 \%$ \\
\hline Throughput & $97 \%$ & $98 \%$ & $98 \%$ & $96 \%$ \\
\hline \multirow[t]{2}{*}{ Target Load } & $=150 \%$ & & & \\
\hline & $\begin{array}{c}\mathrm{ECN} \\
-------\end{array}$ & $\begin{array}{c}\mathrm{ECN}+ \\
-------\end{array}$ & $\begin{array}{c}\mathrm{ECN}+/ \text { Wait } \\
-------\end{array}$ & $\begin{array}{c}\mathrm{ECN}+/ \operatorname{TryOnce} \\
---------\end{array}$ \\
\hline Dropped & 130,007 & 251,856 & 326,845 & 133,603 \\
\hline Marked & 63,066 & 146,757 & 147,239 & 66,444 \\
\hline Loss rate & $42.5 \%$ & $61.3 \%$ & $67.3 \%$ & $31.7 \%$ \\
\hline Throughput & $93 \%$ & $99 \%$ & $99 \%$ & $94 \%$ \\
\hline
\end{tabular}




\begin{tabular}{|c|c|c|c|c|c|c|c|c|c|c|c|}
\hline TIME: & 10 & 100 & 200 & 300 & 400 & 500 & 1000 & 2000 & 3000 & 4000 & 5000 \\
\hline ECN : & 0.00 & 0.05 & 0.18 & 0.42 & 0.70 & 0.86 & 0.88 & 0.88 & 0.88 & 0.98 & 0.98 \\
\hline $\mathrm{ECN}+$ : & 0.00 & 0.06 & 0.20 & 0.45 & 0.78 & 0.96 & 1.00 & 1.00 & 1.00 & 1.00 & 1.00 \\
\hline Wait: & 0.00 & 0.05 & 0.18 & 0.40 & 0.68 & 0.84 & 0.96 & 1.00 & 1.00 & 1.00 & 1.00 \\
\hline Once: & 0.00 & 0.05 & 0.18 & 0.40 & 0.71 & 0.88 & 0.96 & 0.97 & 0.97 & 0.99 & 0.99 \\
\hline arget & Load & $=110$ & $0 \%:$ & & & & & & & & \\
\hline TIME: & 10 & 100 & 200 & 300 & 400 & 500 & 1000 & 2000 & 3000 & 4000 & 5000 \\
\hline $\mathrm{ECN}$ : & 0.00 & 0.03 & 0.13 & 0.29 & 0.52 & 0.66 & 0.69 & 0.69 & 0.69 & 0.91 & 0.91 \\
\hline $\mathrm{ECN}+$ : & 0.00 & 0.05 & 0.17 & 0.36 & 0.66 & 0.88 & 0.98 & 0.99 & 1.00 & 1.00 & 1.00 \\
\hline Wait: & 0.00 & 0.02 & 0.08 & 0.20 & 0.35 & 0.47 & 0.76 & 0.98 & 1.00 & 1.00 & 1.00 \\
\hline Once: & 0.00 & 0.05 & 0.15 & 0.32 & 0.58 & 0.75 & 0.88 & 0.90 & 0.90 & 0.97 & 0.97 \\
\hline Target & Load & $=125$ & & & & & & & & & \\
\hline TIME : & 10 & 100 & 200 & 300 & 400 & 500 & 1000 & 2000 & 3000 & 4000 & 5000 \\
\hline $\mathrm{ECN}$ : & 0.00 & 0.03 & 0.10 & 0.22 & 0.40 & 0.52 & 0.56 & 0.56 & 0.57 & 0.82 & 0.82 \\
\hline $\mathrm{ECN}+$ : & 0.00 & 0.03 & 0.14 & 0.27 & 0.49 & 0.70 & 0.96 & 0.99 & 0.99 & 0.99 & 1.00 \\
\hline Wait: & 0.00 & 0.00 & 0.03 & 0.07 & 0.12 & 0.18 & 0.50 & 0.94 & 0.99 & 0.99 & 1.00 \\
\hline Once: & 0.00 & 0.04 & 0.13 & 0.28 & 0.51 & 0.66 & 0.81 & 0.84 & 0.84 & 0.94 & 0.94 \\
\hline Target & Load & $=150$ & & & & & & & & & \\
\hline TIME : & & 100 & 200 & 300 & 400 & 500 & 1000 & 2000 & 3000 & 4000 & 5000 \\
\hline ECN : & 0.00 & 0.02 & 0.07 & 0.15 & 0.28 & 0.38 & 0.42 & 0.42 & 0.43 & 0.67 & 0.68 \\
\hline $\mathrm{ECN}+$ : & 0.00 & 0.00 & 0.00 & 0.00 & 0.01 & 0.05 & 0.68 & 0.83 & 0.95 & 0.97 & 0.98 \\
\hline Wait: & 0.00 & 0.00 & 0.00 & 0.00 & 0.00 & 0.00 & 0.10 & 0.62 & 0.83 & 0.93 & 0.97 \\
\hline Once: & 0.00 & 0.03 & 0.11 & 0.24 & 0.42 & 0.56 & 0.71 & 0.75 & 0.75 & 0.88 & 0.88 \\
\hline 4 & $\begin{array}{l}\text { The } \\
\text { tim } \\
\text { Kby } \\
\text { pac }\end{array}$ & $\begin{array}{l}\text { cum } \\
\text { s, } \\
\text { es, } \\
\text { ets } \\
\text { p: / }\end{array}$ & $\begin{array}{r}r \text { s } \\
10 \\
\text { (th } \\
\text { WW. }\end{array}$ & & ons & $\begin{array}{l}\text { ion } \mathrm{fu} \\
\text { ith ar } \\
\text { RED in }\end{array}$ & a & age & $\begin{array}{l}\mathrm{DF}) \\
\text { flow } \\
\text { ode. }\end{array}$ & $\begin{array}{l}\text { or tr } \\
\text { size } \\
\text { queue }\end{array}$ & $\begin{array}{l}\text { ansf } \\
\text { of } 3 \\
\text { in }\end{array}$ \\
\hline
\end{tabular}

A.2. Simulations with RED in Byte Mode

Table 5 below shows simulations with RED in byte mode and the queue in bytes. There is no significant increase in aggregate congestion with the use of ECN+, ECN+/Wait, or ECN+/TryOnce.

However, unlike the simulations with RED in packet mode, the simulations with RED in byte mode show little benefit from the use of ECN+ or ECN+/Wait, in that the packet marking rate with ECN+ or 
ECN+/Wait is not much different than the packet marking rate with Standard ECN. This is because with RED in byte mode, small packets like SYN/ACK packets are rarely dropped or marked -- that is, there is no drawback from the use of ECN+ in these scenarios, but not much need for ECN+ either, in a scenario where small packets are unlikely to be dropped or marked.

\begin{tabular}{|c|c|c|c|c|}
\hline & ECN & $\begin{array}{c}\mathrm{ECN}+ \\
------\end{array}$ & $\mathrm{ECN}+/$ Wait & $\mathrm{ECN}+/ \operatorname{TryOnce}$ \\
\hline Dropped & 766 & 446 & 427 & 408 \\
\hline Marked & 32,683 & 34,289 & 33,412 & 31,892 \\
\hline Loss rate & $0.05 \%$ & $0.03 \%$ & $0.03 \%$ & $0.03 \%$ \\
\hline Throughput & $81 \%$ & $81 \%$ & $81 \%$ & $81 \%$ \\
\hline \multirow[t]{3}{*}{ Target Load } & $=110 \%$ & & & \\
\hline & ECN & $\mathrm{ECN}+$ & $\mathrm{ECN}+/$ Wait & $\mathrm{ECN}+/$ TryOnce \\
\hline & ------- & ------- & ------- & ---------- \\
\hline Dropped & 2,496 & 2,110 & 1,733 & 2,020 \\
\hline Marked & 220,573 & 258,696 & 230,955 & 214,604 \\
\hline Loss rate & $0.15 \%$ & $0.13 \%$ & $0.11 \%$ & $0.11 \%$ \\
\hline Throughput & $92 \%$ & $91 \%$ & $92 \%$ & $92 \%$ \\
\hline \multirow[t]{3}{*}{ Target Load } & $=125 \%$ & & & \\
\hline & $\mathrm{ECN}$ & $\mathrm{ECN}+$ & $\mathrm{ECN}+/$ Wait & $\mathrm{ECN}+/$ TryOnce \\
\hline & ------- & ------- & ------- & ---------- \\
\hline Dropped & 20,032 & 13,555 & 13,979 & 16,918 \\
\hline Marked & 725,165 & 726,992 & 726,823 & 615,235 \\
\hline Loss rate & $1.11 \%$ & $0.76 \%$ & $0.78 \%$ & $0.66 \%$ \\
\hline Throughput & $95 \%$ & $95 \%$ & $95 \%$ & $96 \%$ \\
\hline \multirow[t]{3}{*}{ Target Load } & $=150 \%$ & & & \\
\hline & $\mathrm{ECN}$ & $\mathrm{ECN}+$ & $\mathrm{ECN}+/$ Wait & $\mathrm{ECN}+/ \operatorname{TryOnce}$ \\
\hline & ------- & ------- & ------- & ---------- \\
\hline Dropped & 484,251 & 483,847 & 507,727 & 600,737 \\
\hline Marked & 865,905 & 872,254 & 873,317 & 818,451 \\
\hline Loss rate & $19.09 \%$ & $19.13 \%$ & $19.71 \%$ & $12.66 \%$ \\
\hline Throughput & $99 \%$ & $98 \%$ & $99 \%$ & $99 \%$ \\
\hline
\end{tabular}

Table 5: Simulations with an average flow size of 3 kbytes, a 100 Mbps link, RED in byte mode, queue in bytes 


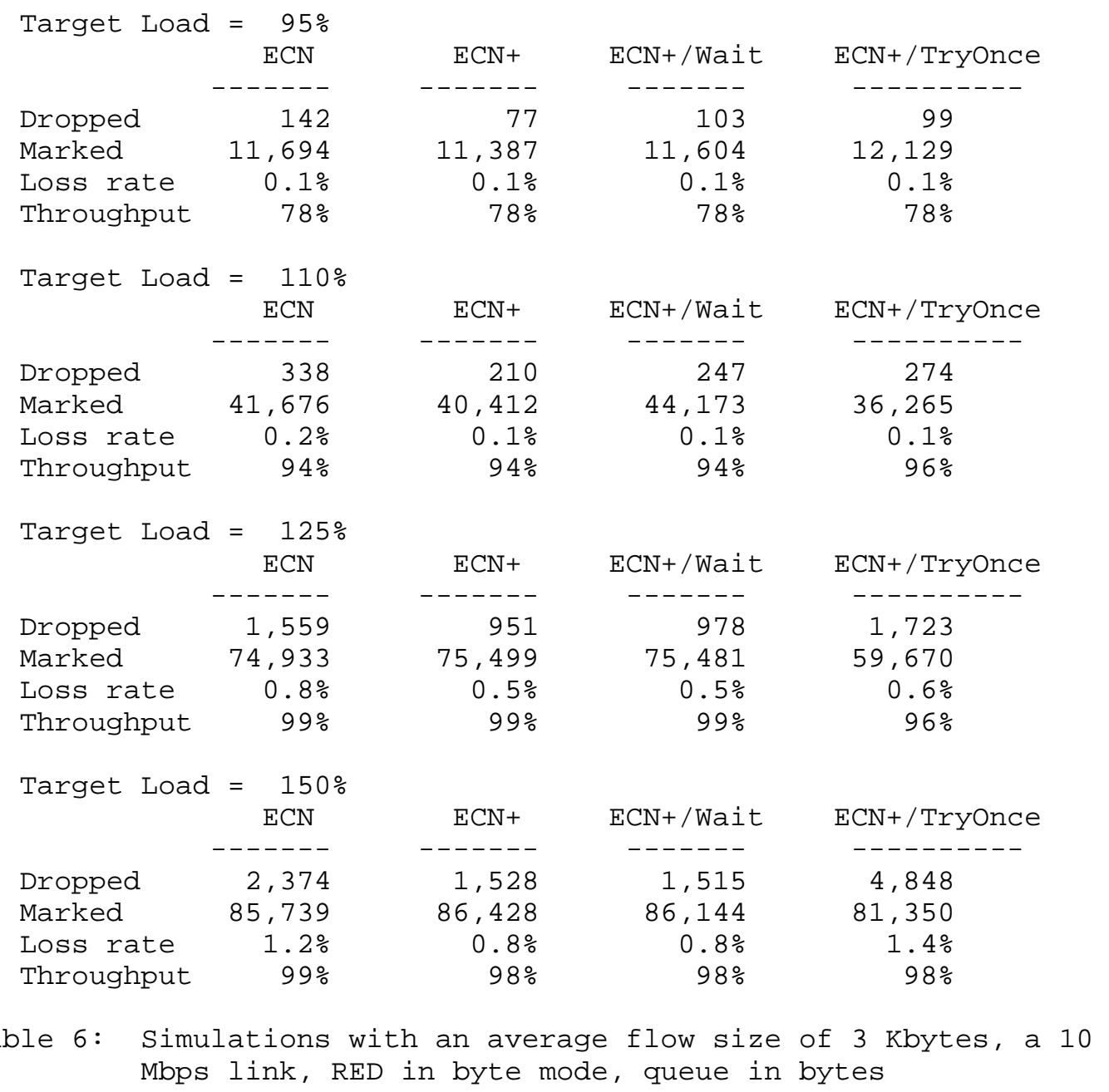


Appendix B. Issues of Incremental Deployment

In order for TCP node B to send a SYN/ACK packet as ECN-Capable, node $B$ must have received an ECN-setup SYN packet from node A. However, it is possible that node A supports ECN, but either ignores the CE codepoint on received SYN/ACK packets, or ignores SYN/ACK packets with the ECT or CE codepoint set. If the TCP initiator ignores the $\mathrm{CE}$ codepoint on received SYN/ACK packets, this would mean that the TCP responder would not respond to this congestion indication. However, this seems to us an acceptable cost to pay in the incremental deployment of ECN-Capability for TCP'S SYN/ACK packets. It would mean that the responder would not reduce the initial congestion window from two, three, or four segments down to one segment, as it should, and would not sent a non-ECN-Capable SYN/ACK packet to complete the SYN exchange. However, the TCP end nodes would still respond correctly to any subsequent CE indications on data packets later on in the connection.

Figure 4 shows an interchange with the SYN/ACK packet ECN-marked, but with the ECN mark ignored by the TCP originator.

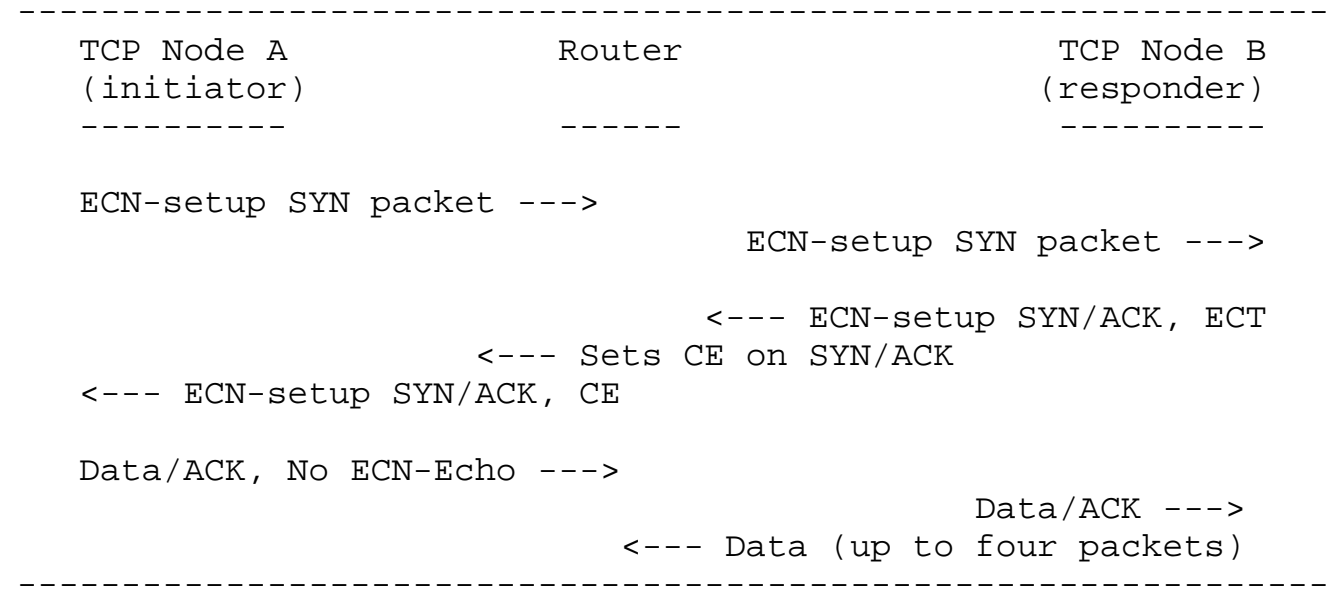

Figure 4: SYN exchange with the SYN/ACK packet marked, but with the ECN mark ignored by the TCP initiator

Thus, to be explicit, when a TCP connection includes an initiator that supports ECN but *does not* support ECN-Capability for SYN/ACK packets, in combination with a responder that *does* support ECNCapability for SYN/ACK packets, it is possible that the ECN-Capable SYN/ACK packets will be marked rather than dropped in the network, and that the responder will not learn about the ECN mark on the SYN/ACK packet. This would not be a problem if most packets from the 
responder supporting ECN for SYN/ACK packets were in long-lived TCP connections, but it would be more problematic if most of the packets were from TCP connections consisting of four data packets, and the TCP responder for these connections was ready to send its data packets immediately after the SYN/ACK exchange. Of course, with *severe* congestion, the SYN/ACK packets would likely be dropped rather than ECN-marked at the congested router, preventing the TCP responder from adding to the congestion by sending its initial window of four data packets.

It is also possible that in some older TCP implementation, the initiator would ignore arriving SYN/ACK packets that had the ECT or CE codepoint set. This would result in a delay in connection setup for that TCP connection, with the initiator re-sending the SYN packet after a retransmission timeout. We are not aware of any TCP implementations with this behavior.

One possibility for coping with problems of backwards compatibility would be for TCP initiators to use a TCP flag that means "I understand ECN-Capable SYN/ACK packets". If this document were to standardize the use of such an "ECN-SYN" flag, then the TCP responder would only send a SYN/ACK packet as ECN-Capable if the incoming SYN packet had the "ECN-SYN" flag set. An ECN-SYN flag would prevent the backwards compatibility problems described in the paragraphs above.

One drawback to the use of an ECN-SYN flag is that it would use one of the four remaining reserved bits in the TCP header for a transient backwards compatibility problem. This drawback is limited by the fact that the "ECN-SYN" flag would be defined only for use with ECNsetup SYN packets; that bit in the TCP header could be defined to have other uses for other kinds of TCP packets.

Factors in deciding not to use an ECN-SYN flag include the following:

(1) The limited installed base: At the time that this document was written, the TCP implementations in Microsoft Vista and Mac OS X included ECN, but ECN was not enabled by default [SBT07]. Thus, there was not a large deployed base of ECN-Capable TCP implementations. This limits the scope of any backwards compatibility problems.

(2) Limits to the scope of the problem: The backwards compatibility problem would not be serious enough to cause congestion collapse; with severe congestion, the buffer at the congested router will overflow, and the congested router will drop rather than ECN-mark 
arriving SYN packets. Some active queue management mechanisms might switch from packet-marking to packet-dropping in times of high congestion before buffer overflow, as recommended in section 19.1 of RFC 3168 [RFC3168]. This helps to prevent congestion collapse problems with the use of ECN.

(3) Detection of and response to backwards-compatibility problems: A TCP responder such as a web server can't differentiate between a SYN/ACK packet that is not ECN-marked in the network, and a SYN/ACK packet that is ECN-marked, but where the ECN mark is ignored by the TCP initiator. However, a TCP responder * Can* detect if a SYN/ACK packet is sent as ECN-capable and not reported as ECN-marked, but data packets are dropped or marked from the initial window of data. We will call this scenario "initial-window-congestion". If a web server frequently experienced initial-window-congestion (without SYN/ACK congestion), then the web server *might* be experiencing backwards compatibility problems with ECN-Capable SYN/ACK packets, and could respond by not sending SYN/ACK packets as ECN-Capable.

Normative References

[RFC793] Postel, J., "Transmission Control Protocol", STD 7, RFC 793, September 1981 .

[RFC2119] Bradner, S., "Key words for use in RFCs to Indicate Requirement Levels", BCP 14, RFC 2119, March 1997.

[RFC2988] Paxson, V. and M. Allman, "Computing TCP's Retransmission Timer", RFC 2988, November 2000.

[RFC3168] Ramakrishnan, K., Floyd, S., and D. Black, "The Addition of Explicit Congestion Notification (ECN) to IP", RFC 3168, September 2001 .

Informative References

[ECN+] A. Kuzmanovic, The Power of Explicit Congestion Notification, SIGCOMM 2005.

[ECN-SYN] ECN-SYN web page with simulation scripts, http://www.icir.org/floyd/ecn-syn. 
[F07] S. Floyd, "[BEHAVE] Response of firewalls and middleboxes to TCP SYN packets that are ECN-Capable?", August 2, 2007 , email to the BEHAVE mailing list, http://www1.ietf.org/ mail-archive/web/behave/current/msg02644.html.

[Kelson00] Dax Kelson, "8\% of the Internet unreachable!", September 10, 2000, email to the Linux kernel mailing list, http://lkml.indiana.edu/hypermail/linux/kernel/ $0009.1 / 0329$. html.

[L08] A. Landley, "Re: [tcpm] I-D Action:draft-ietf-tcpmecnsyn-06.txt", August 24, 2008, email to the tcpm mailing list, http://www.ietf.org/ mail-archive/web/tcpm/current/msg03988.html.

[MAF05] A. Medina, M. Allman, and S. Floyd, "Measuring the Evolution of Transport Protocols in the Internet", ACM CCR, April 2005.

[PI] C. Hollot, V. Misra, W. Gong, and D. Towsley, "On Designing Improved Controllers for AQM Routers Supporting TCP Flows", April 1998.

[RED] Floyd, S., and Jacobson, V., "Random Early Detection gateways for Congestion Avoidance", IEEE/ACM Transactions on Networking, V.1 N.4, August 1993.

[REM] S. Athuraliya, V. H. Li, S. H. Low and Q. Yin, "REM: Active Queue Management", IEEE Network, May 2001.

[RFC2309] Braden, B., Clark, D., Crowcroft, J., Davie, B., Deering, S., Estrin, D., Floyd, S., Jacobson, V., Minshall, G., Partridge, C., Peterson, L., Ramakrishnan, K., Shenker, S., Wroclawski, J., and L. Zhang, "Recommendations on Queue Management and Congestion Avoidance in the Internet", RFC 2309, April 1998.

[RFC2581] Allman, M., Paxson, V., and W. Stevens, "TCP Congestion Control", RFC 2581, April 1999.

[RFC3042] Allman, M., Balakrishnan, H., and S. Floyd, "Enhancing TCP's Loss Recovery Using Limited Transmit", RFC 3042, January 2001 .

[RFC3360] Floyd, S., "Inappropriate TCP Resets Considered Harmful", BCP 60, RFC 3360, August 2002 . 
[RFC3390] Allman, M., Floyd, S., and C. Partridge, "Increasing TCP'S Initial Window", RFC 3390, October 2002 .

[RFC4987] Eddy, W., "TCP SYN Flooding Attacks and Common Mitigations", RFC 4987, August 2007.

[SCJO01] F. Smith, F. Campos, K. Jeffay, and D. Ott, "What TCP/IP Protocol Headers Can Tell us about the Web", SIGMETRICS, June 2001.

[SYN-COOK] Dan J. Bernstein, SYN cookies, 1997, see also <http://cr.yp.to/syncookies.html>.

[SBT07] M. Sridharan, D. Bansal, and D. Thaler, "Implementation Report on Experiences with Various TCP RFCs", Presentation in the TSVAREA, IETF 68, March 2007. http: //www3. ietf.org/proceedings/07mar/slides/tsvarea$3 /$ sld6.htm.

[Tools] S. Floyd, Ed., and E. Kohler, Ed., "Tools for the Evaluation of Simulation and Testbed Scenarios", Work in Progress, February 2008 . 


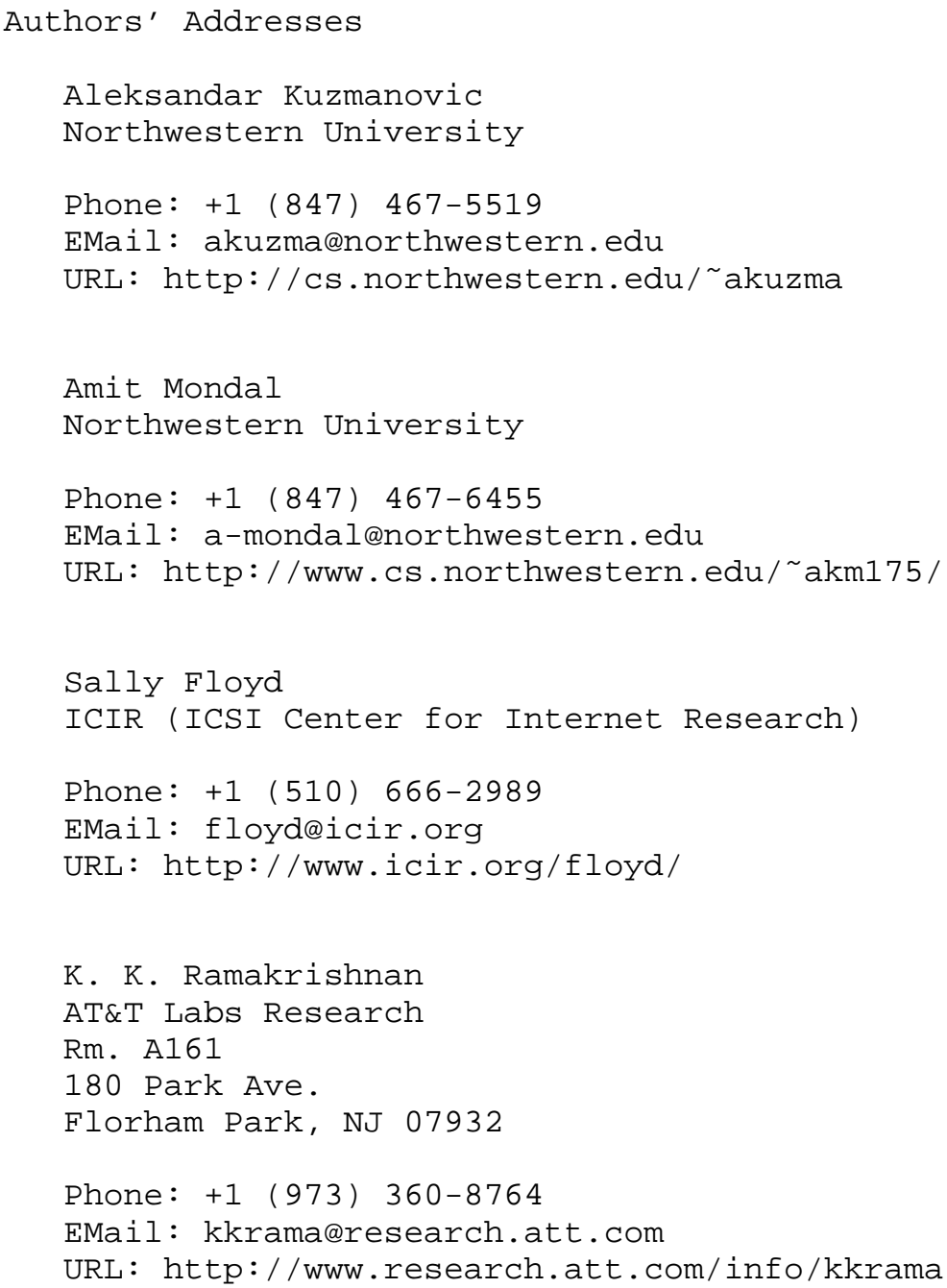

